\title{
Randomized clinical trial to evaluate the efficacy of a 5-day ceftiofur hydrochloride intramammary treatment on nonsevere gram-negative clinical mastitis
}

\author{
Y. H. Schukken, ${ }^{* 1}$ G. J. Bennett, ${ }^{*}$ M. J. Zurakowski, ${ }^{*}$ H. L. Sharkey, ${ }^{*}$ B. J. Rauch, ${ }^{\star}$ M. J. Thomas, $\dagger$ \\ B. Ceglowski,‡ R. L. Saltman,§ N. Belomestnykh, ${ }^{*}$ and R. N. Zadoks*\# \\ ${ }^{*}$ Quality Milk Production Services, Department of Population Medicine and Diagnostic Sciences, College of Veterinary Medicine, Cornell \\ University, Ithaca, NY 14853 \\ †Countryside Veterinary Clinic, Lowville, NY 13367 \\ ‡Rupert Veterinary Clinic, Rupert, VT 05768 \\ §Pfizer Animal Health, New York, NY 10017 \\ \#Moredun Research Institute, Penicuik, UK and Royal (Dick) School of Veterinary Studies, University of Edinburgh, Roslin, UK
}

\section{ABSTRACT}

The objective of this study was to evaluate the efficacy of intramammary treatment with ceftiofur hydrochloride of nonsevere, clinical coliform mastitis. One hundred four cases on 5 farms met the enrollment criteria for the study. Escherichia coli was the most common coliform species identified in milk samples from cows with mild to moderate clinical mastitis, followed by Klebsiella spp. and Enterobacter spp. At enrollment, a milk sample from the affected quarter was taken and used for on-farm culture or submitted to the laboratory. For cows in the treatment group, treatment was initiated with ceftiofur hydrochloride via intramammary infusion at 24-h intervals for $5 \mathrm{~d}$ according to label standards. Cows in the control group did not receive treatment. Culture results were available on the day after enrollment and only cows with coliform mastitis continued in the treatment and untreated control groups. Bacteriological cure was defined based on 2 posttreatment milk samples. Molecular typing was used for final definition of bacteriological cure. Treatment of nonsevere clinical gram-negative mastitis with ceftiofur hydrochloride resulted in a significant increase in bacteriological cure compared with nontreated controls in animals infected with E. coli or Klebsiella spp. Treated animals clinically improved significantly more compared with control cows. No significant differences were observed between treated and control animals in milk production or linear score before or after clinical mastitis. Treated animals left the study less frequently compared with control animals.

Key words: gram-negative mastitis, ceftiofur hydrochloride, Escherichia coli, Klebsiella

Received February 18, 2011.

Accepted August 27, 2011.

${ }^{1}$ Corresponding author: yschukken@cornell.edu

\section{INTRODUCTION}

Mastitis is widespread in dairy herds. It is the most costly disease to animal agriculture in the United States and throughout much of the world. It affects animal welfare and results in decreased milk yield, increased cost of milk production, and reduced milk quality (Barkema et al., 1998; Gröhn et al., 2004). In dairy cows, mastitis is usually caused by bacteria or other microorganisms that enter the mammary gland. Gram-negative bacteria, mostly coliforms (Escherichia coli, Klebsiella spp., and Enterobacter spp.), may cause a high proportion of all clinical mastitis (CM) cases (Barkema et al., 1998; Olde Riekerink et al., 2008; Breen et al., 2009; Nam et al., 2009). In published field studies, 15 to $25 \%$ of cows in well-managed herds are diagnosed annually with CM caused by coliforms (Erskine et al., 1988; Hogan and Smith, 2003). The most common coliform species reported to cause CM are E. coli and Klebsiella spp. (Todhunter et al., 1991; Erskine et al., 2002a,b; Munoz et al., 2007). Klebsiella spp. appear to be particularly important in North American herds.

Results from experimental studies and field studies on mastitis caused by $E$. coli or other coliforms have been equivocal. Experimental challenge studies with $E$. coli conducted by Pyörälä et al. (1994) with 6 cows per treatment group showed no positive effect of treatment with local colistin sulfate or parenteral trimethroprim sulfadiazine. Using an experimental $E$. coli challenge and 2 cows per treatment group, Rantala et al. (2002) observed a positive effect of parenteral enrofloxacin administration on milk yield but not on clinical signs or survival. In an experimental challenge study with 6 cows per treatment group, Hoeben et al. (2000) also demonstrated a beneficial effect of enrofloxacin on milk yield but not on general clinical signs or milk score after experimental challenge with E. coli. Systemically administered danofloxacin had a positive effect on milk 
yield and on bacteriological results and systemic and local clinical signs in a negative controlled E. coli experimental challenge trial (Poutrel et al., 2008). In contrast to experimental studies, a field study of natural E. coli mastitis did not show a beneficial effect of enrofloxacin (Suojala et al., 2010). Conflicting results have also been obtained for cephalosporins. A positive controlled experimental challenge trial with a total of 47 cows showed that parenteral administration of cefquinome resulted in more clinical and bacterial cure than use of intramammary amoxicillin and cloxacillin (Shpigel et al., 1997). A field study with 29 treated cases and 27 untreated controls of naturally occurring severe clinical mastitis caused by coliform organisms showed that intramuscular treatment with ceftiofur reduced the risk of death or culling (Erskine et al., 2002a). In a field study of 33 cows with mild clinical mastitis cases that were predominantly caused by E. coli, however, intramuscular ceftiofur treatment in combination with intramammary cephapirin treatment did not have beneficial effects (Wenz et al., 2005) compared with intramammary treatment alone. The observation that systemic ceftiofur treatment does not improve the cure of intramammary infection is not unexpected because systemic ceftiofur treatment does not result in appreciable titers in milk in cows without systemic clinical signs (Erskine et al. 1995). When in the Wenz et al. (2005) study, the intramammary cephapirin treatment alone was compared with an intramammary treatment with pirlimycin (an antibiotic not efficient against coliform bacteria), a difference in bacteriological cure of coliforms of 18 percentage points was observed (50\% vs. $32 \%$, respectively). Because of the small number of animals in the study, however, this difference was not statistically significant $(P=0.2)$.

Treatment of E. coli CM with intramammary ceftiofur hydrochloride received FDA approval in the United States based on a field study showing $86 \%$ bacteriological cure in treated cows versus $46 \%$ bacteriological cure in control cows (FDA, 2005). However, the numbers of cows treated in each of the treatment arms of the study were relatively small, with 14 and 13 cows in the treatment and control groups, respectively. No efficacy data of ceftiofur against Klebsiella spp. mastitis are currently available even though Klebsiella mastitis is emerging as an important cause of clinical mastitis on dairy farms, particularly in the United States (Munoz et al., 2007). Generally, both E. coli and Klebsiella spp. isolated from bovine mastitis cases have been found to be susceptible in vitro to third-generation cephalosporins (Srinivasan et al., 2007; Bengtsson et al., 2009; Nam et al., 2009).

The objective of this study was to evaluate the efficacy of intramammary treatment of nonsevere, clinical coliform mastitis with ceftiofur hydrochloride.
Particularly, the bacteriological cure rates and clinical symptoms of cows treated with ceftiofur hydrochloride were compared with those of control cows that were not treated with antimicrobials (negative controls).

\section{MATERIALS AND METHODS}

\section{Farm Inclusion Criteria}

Inclusion criteria for farms included (1) having at least 500 cows milking on a given day, (2) availability of reliable health records, and (3) access to culture results from milk samples of $\mathrm{CM}$ cases within $1 \mathrm{~d}$ of sample collection. Preferably, farms that made use of DHIA services, including monthly SCC, were included. Health records included calving, treatment, vaccination, fertility, and culling data. Herds were allowed to vaccinate against coliform mastitis (J5) as long as all cows received the same vaccination program. Access to culture results within $1 \mathrm{~d}$ of sample collection was achieved through use of on-farm culture systems or daily sample pick-up through the Quality Milk Production Services (QMPS) program. Farms included in the study (A to E) are described in Table 1.

\section{Study Design and Quality Assurance}

Sample size was calculated using the following assumptions: one-sided significance level, $\alpha$, at 0.05 , power, $1-\beta$, at 0.8 assumed cure rate in the controls of 0.5 and in the treated group of 0.7 or higher. The estimated study size was 50 cases per treatment group or approximately 100 cows to be enrolled in the trial. A one-sided significance level was used because realistically no negative effect of treatment was expected. The study was conducted as a randomized block design with cows within herds randomly assigned to the treatment or control group. Randomization was based on ear tag number. For each herd, cows with odd ear tag numbers were assigned to the treatment group and cows with even ear tag numbers were assigned to the control group. Treatment instructions were in numbered binders. Intramammary treatments were administered by trained farm personnel. Clinical assessments were performed by trained farm personnel before the first treatment, once a day thereafter until the completion of treatment, and by QMPS personnel when follow-up samples were collected. All clinical recordings, treatments, and follow-up measurements were recorded on case record forms and signed off by the person making the observations. All binders were accounted for on the dairy. Data were entered electronically by one of the authors (HLS) and double-checked for accuracy. Copies of the computerized herd record-keeping sys- 
Table 1. Basic description upon entering the study of 104 enrolled coliform mastitis cases in 5 farms (A to E, all in New York State) ${ }^{1}$

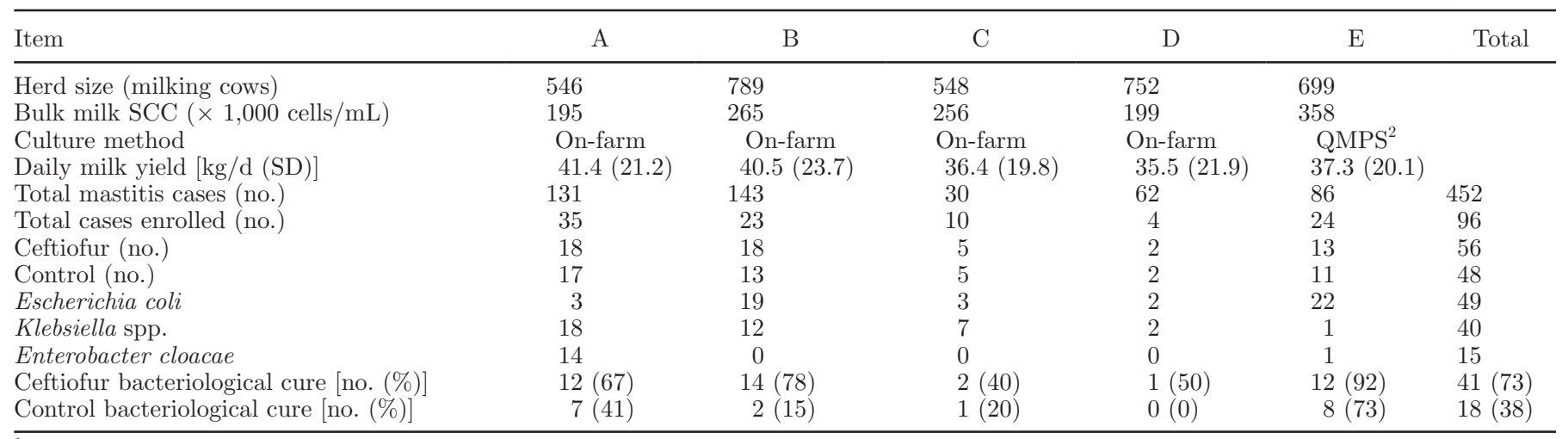

${ }^{1}$ Culture results are from the pretreatment milk sample.

${ }^{2}$ QMPS $=$ Quality Milk Production Services.

tem (DairyComp305, Valley Ag Software, Tulare, CA) were collected regularly. Milk production and SCC were taken from DHIA test-days and extracted from the DairyComp305 records. If available, data from 3 test-days were obtained immediately before and after the case of clinical mastitis. After completion of the treatment, and at equivalent time points for untreated control cows, 2 follow-up milk samples were collected by QMPS personnel at $7 \pm 2 \mathrm{~d}$ and $14 \pm 2 \mathrm{~d}$ after the last treatment. Follow-up samples were taken at 7 and $14 \mathrm{~d}$ after the last treatment to accommodate the relatively short duration of E. coli IMI (Pyörälä et al., 1994; Hoeben et al., 2000), thereby preventing the risk of new infections to distort the observed cure risks. All mastitis samples and follow-up samples were submitted to a QMPS laboratory, including samples that had been used for on-farm culture.

\section{Cow Inclusion Criteria and Treatment}

Cows were eligible for inclusion in the study if they had not been treated with antibiotics in the last 14 $\mathrm{d}$ and did not show severe teat lesions due to recent trauma. Cows were eligible for inclusion from the day of calving (inclusive) until $25 \mathrm{~d}$ before dry-off. A cow was only eligible to be included once in the study. Cows were enrolled when they presented with signs of nonsevere (mild or moderate) CM in one quarter. At enrollment, a milk sample from the affected quarter was taken using standard aseptic technique (NMC, 1999), used for on-farm culture, and submitted to QMPS. Cows only continued in the study if the on-farm or QMPS culture result at $24 \mathrm{~h}$ after inclusion showed the presence of coliform organisms. Eventually, cows were only included in this analysis when the enrollment sample was confirmed to be coliform positive at the QMPS laboratory.
For cows in the treatment group, treatment with ceftiofur hydrochloride $(125 \mathrm{mg}$ in $10 \mathrm{~mL}$, Spectramast LC, Pfizer Animal Health, New York, NY) was initiated via intramammary infusion at 24-h intervals for $5 \mathrm{~d}$ according to label standards. Cows in the control group did not receive treatment on the day of enrollment. On the day after enrollment, culture results were available and only cows with coliform mastitis continued in the treatment and untreated control groups. All other animals (e.g., animals with gram-positive mastitis or culture negative milk samples) were excluded from the study and could be treated at the discretion of the herd manager or veterinarian. The study was approved by the Cornell University Institutional Animal Care and Use Committee.

\section{Clinical Scoring System}

The clinical scoring system described and evaluated by Wenz et al. (2001, 2006) was used to classify CM severity. A score of mild was assigned when the milk was abnormal (flakes, watery, clots, or bloody) and no other local or systemic signs of inflammatory disease were observed. A score of moderate was assigned if the milk was abnormal, swelling or pain of the affected mammary gland was noted, and only one of the systemic signs of inflammatory disease defined below was observed. A score of severe was assigned when the milk was grossly abnormal, swelling or pain of the affected gland was noted, and at least 2 of the following systemic disease signs were seen: rectal temperature $\geq 39.5^{\circ} \mathrm{C}$, moderate to marked enophthalmos ( $>3 \mathrm{~mm})$, or marked depression defined as inappetence or not able to stand or both (Wenz et al., 2001, 2006). For analysis of the data, each milk, mammary, or systemic sign described above was coded as present (1) or absent (0), resulting in a milk 
score from 0 to 4 , a mammary score from 0 to 2 , and a systemic score from 0 to 3 . The total clinical score then ranged between 0 and 9 .

Cows were considered clinically cured if all posttreatment scores were completely normal $($ score $=0)$. Any abnormality at either of the 2 follow-up evaluations was considered a clinical noncure. Because complete absence of any clinical signs within $14 \mathrm{~d}$ after the end of treatment may be difficult to achieve from any treatment, we also evaluated clinical improvement of the included cows. A cow was defined as clinically improved if the clinical score at the 14-d posttreatment evaluation was $50 \%$ or less of the score obtained at the initial diagnosis of clinical mastitis. That is, the animal had improved her clinical score by at least $50 \%$.

\section{Bacteriological Identification and Definition of Infection}

A quarter was considered infected with a coliform organism and included in the analysis if the milk sample taken immediately before enrollment was confirmed to be coliform positive at the QMPS laboratory. Approximately $0.01 \mathrm{~mL}$ of milk was streaked on trypticase soy agar containing 5\% sheep blood and $0.1 \%$ esculin (PML Microbiologicals, Mississauga, ON, Canada), and plates were incubated aerobically at $37^{\circ} \mathrm{C}$ for $48 \mathrm{~h}$. After observation of colony morphology and hemolytic patterns on blood agar, isolates were examined further by means of $3 \% \mathrm{KOH}$, Gram staining of organisms, catalase and oxidase testing, and additional biochemical and metabolic evaluations as needed. Gram-negative organisms were identified by colony morphology on MacConkey's agar (National Mastitis Council, 1999). Lactose-positive, citrate-negative colonies surrounded by bile-salt precipitate on MacConkey agar were considered E. coli, and yellow or pink mucoid colonies without precipitate and motility negative were considered Klebsiella spp. Klebsiella pneumoniae and Klebsiella oxytoca were differentiated using indol. For isolates that were phenotypically identified as belonging to the genus Enterobacter, species identity was determined based on rpoB sequencing (Munoz et al., 2007). Strain typing was used to compare pre- and posttreatment isolates if the same bacterial species was observed before and after treatment of a quarter. Per quarter, between 1 and 5 isolates were selected depending on the number of isolates available before and after treatment. In several cows, quarters were also sampled during treatment, and isolates obtained from these samples were evaluated using molecular strain typing. Strain typing was also used for comparison across quarters within bacterial species within herds. Strain typing was performed by means of random amplified polymorphic DNA (RAPD)-PCR
(Munoz et al., 2007). Reproducibility of RAPD banding patterns was evaluated using an arbitrary selection of 18 isolates for which lysates were prepared on 2 or 3 occasions, 3 wk to 10 mo apart.

Bacteriological cure was defined based on culture results from 2 posttreatment samples and RAPD typing. Both posttreatment samples had to be present and the coliform species identified in the pretreatment sample had to be absent from both posttreatment samples. When the same bacterial species was identified but RAPD typing revealed a different strain pretreatment and posttreatment, the case was defined as a bacteriological cure followed by a new infection. All statistical analyses were performed in SAS software (version 9.2, SAS Institute Inc., Cary, NC).

\section{Statistical Analysis}

Data were initially analyzed using descriptive statistics, and descriptive statistics in treatment and control groups were compared using a $t$-test. Outliers and missing data were further investigated. Cows did not necessarily have complete SCC and milk production data before or after treatment because some cows had mastitis in early lactation and some cows were dried-off or culled before 2 or 3 test-days had passed. For these reasons, analysis of production and cell count data was done on a subgroup of animals. We defined the subgroup for test-day analysis as cows that had at least 2 pretreatment milk production and SCC data points and 2 posttreatment milk production and SCC data points. Milk production and SCC data [after transformation to linear score (LS) using the formula LS = $\log _{2}(\mathrm{SCC} / 100)+3$, where SCC is expressed in units of 1,000 cells $/ \mathrm{mL}$ ] were analyzed using a linear mixed model. The model used for this analysis was

$$
\begin{gathered}
\text { Milk }(\text { or LS })=\text { Lactation + DIM + Herd } \\
+ \text { Pathogen + TRMastitis + treatment + Re, }
\end{gathered}
$$

where Milk or LS is the test-day milk yield $(\mathrm{kg})$ or linear score, lactation is the lactation number categorized in 3 categories $(1,2$, and $3+$ ), DIM is days in milk in ten 30-d categories, Herd is a set of indicator variables indicating herd of origin, Pathogen is a set of indicator variables indicating the causal bacterial species, TRMastitis is a set of indicator variables indicating $-2,-1,1$, or 2 tests relative to the occurrence of clinical mastitis, treatment indicates treatment or control group, and Re is a complex error term consisting of within-cow correlation of test day measurements (REPEATED statement) and a random error term. Two-way interactions were tested and included in the model where significant. 
Clinical cure, clinical improvement, and bacteriological cure were analyzed using logistic regression analysis. Separate analyses were performed for all cases combined and for each bacterial species or genus (E. coli, Klebsiella, Enterobacter) separately. For all regression models, the linear predictor was given by

$$
\begin{gathered}
\text { Logit }(\text { cure })=\text { Lactation }+ \text { DIM }+ \text { Herd } \\
+ \text { Pathogen }+ \text { treatment }+\mathrm{e},
\end{gathered}
$$

where cure is clinical cure, clinical improvement, or bacteriological cure, and Lactation (in 3 categories: 1, 2, and 3+), DIM (in ten 30-d categories), Herd, Pathogen (E. coli, Klebsiella spp., or Enterobacter), and treatment were included as fixed effects, and e was a binomial error term.

Time to exit from the study was defined as the time between entrance into the study and exit from the study. Exit could be due to completion of all data collection (normal exit, treated as censoring in the analysis) or early exit due to culling, death, or intervention by the farmer. Farmer intervention was allowed in the protocol when the clinical status of the cow deteriorated. The farmer indicated the reason for study exit and was free to choose a different or additional treatment for the animal. Time to culling or death was defined as the time between entrance in the study and either culling or death in the lactation where the case of mastitis occurred. Time to event analysis was performed using Kaplan-Meier survival curves and statistically evaluated using Cox proportional hazard models. The linear predictor of the Cox proportional hazards model was

$$
\begin{gathered}
\text { Hazard }(\text { exit })=\text { Base } \times \text { Exp }[\text { Lactation }+ \text { DIM } \\
+ \text { Herd }+ \text { Pathogen }+ \text { treatment }(\text { or cure })]
\end{gathered}
$$

where exit is time to exit, and Lactation, DIM, Herd, and Pathogen were as defined above and included as fixed effects. The effect of treatment versus control and bacteriological cure versus failure was evaluated in 2 separate analyses.

For all outcome variables, treatment was compared with the control group using one-sided significance tests with a $5 \%$ significance level ( $P$-value). All other explanatory variables in the model were evaluated using a 2 -sided significance test with a $P$-value of $5 \%$.

\section{RESULTS}

\section{Cows Enrolled}

In total, 104 cases on 5 farms met the enrollment criteria for the study. Farm characteristics are shown in Table 1 and cow characteristics are shown in Table 2. Mild to moderate $\mathrm{CM}$ due to coliform infections was diagnosed in early, mid, and late lactation. Approximately $40 \%$ of cases occurred in the first 100 DIM and the remainder occurred later in lactation. Average DIM was 136 (SD 97) for treated cows and 140 (SD 88 ) for control cows ( $t$-test, $P=0.82$ ). The majority of affected animals were multiparous cows, with approximately $20 \%$ of cows in first lactation. Average lactation number was 2.5 (SD 1.2) for treated cows and 2.7 (SD 1.4) for control cows ( $t$-test, $P=0.54$ ). Prior to enrollment, milk production of affected cows, as measured at the last test-day before CM, ranged from $<25$ to $>65 \mathrm{~kg} /$ d. Before mastitis, treated animals had an average production of 45.6 (SD 15.6) kg/d and control animals produced $44.3(\mathrm{SD} 10.9) \mathrm{kg} / \mathrm{d}$ ( $t$-test, $P$ $=0.54)$. Treated animals had a pretreatment LS of 3.3 (SD 2.1), whereas control animals had a pretreatment LS of 3.1 (SD 1.9; $t$-test, $P=0.70$ ).

\section{Microbiology and Molecular Typing}

Escherichia coli was the most common coliform species identified in milk samples from cows with mild to moderate CM, followed by Klebsiella spp. and Enterobacter spp. (Table 1). Clear differences were found between herds in distribution of bacterial species or

Table 2. Characteristics of 104 cows with clinical mastitis (CM) cases treated with ceftiofur or untreated (SD in parentheses) controls

\begin{tabular}{lccc}
\hline Parameter & Ceftiofur & Control & $P$-value \\
\hline Cases (no.) & 56 & 48 & \\
Escherichia coli & 29 & 19 & \\
Klebsiella spp. & 20 & 18 & \\
Enterobacter cloacae & 7 & 11 & \\
Cow characteristics & $2.5(1.2)$ & $2.7(1.4)$ & $>0.50$ \\
Lactation number & $135.7(97.2)$ & $139.6(87.6)$ & $>0.80$ \\
Stage of lactation (d) & $45.6(15.6)$ & $44.3(10.9)$ & $>0.50$ \\
Milk production before CM (kg) & $3.3(2.1)$ & $3.1(1.9)$ & $>0.70$ \\
Linear score before CM & $310.4(424.8)$ & $369.1(892.7)$ & \\
SCC before CM $(\times 1,000$ cells $/ \mathrm{mL})$ & & & \\
\hline
\end{tabular}


genera. Herd A showed a predominance of Klebsiella spp. and Enterobacter spp., whereas herd E had predominantly E. coli infections. All Klebsiella isolates were identified as Klebsiella pneumoniae with the exception of one Klebsiella oxytoca in farm B. Because of the vast dominance of Klebsiella pneumoniae in the data, all Klebsiella isolates were analyzed as one group, identified as Klebsiella spp. All Enterobacter isolates that were subjected to $r p o \mathrm{~B}$ sequencing were identified as E. cloacae (99\% species identity based on $100 \%$ coverage of 900-bp fragment). Reproducibility of RAPD banding patterns was excellent, unless lysates had been prepared 10 mo apart, in which case subtle differences in band intensity were observed. To ensure that this did not affect comparisons of RAPD patterns within quarters, cows, or herds, only sets of lysates that were prepared at the same time were used for comparisons. The RAPD patterns for Klebsiella spp. isolates all showed a band of approximately $200 \mathrm{bp}$, and RAPD patterns for E. cloacae isolates all showed a band of approximately $290 \mathrm{bp}$. The RAPD patterns for $E$. coli showed only bands that were larger than 300 bp (Figure 1). Isolates for strain typing were available from 33 of 35 cows in herd A, 28 of 31 cows in herd $\mathrm{B}, 8$ of 10 cows in herd C, 0 of 4 cows in herd D, and 18 of 24 cows in herd E. Of the 17 cows for which strain typing data were not available, 7 were considered treatment failures because they were culled and 8 were considered treatment successes because they were culture negative posttreatment. For 25 animals, cure decisions depended on strain typing data. This included 2 animals for which no strain typing data were available and 2 animals for which only pretreatment strain typing data were available. Of the remaining 21 animals, 17 were considered noncured because the same strain was detected before and after treatment, and 4 animals were considered cured because strains differed before and after treatment (Figure 1). When multiple isolates were available from a quarter before or after treatment, respectively, isolates belonged to the same strain for 44 of 46 episodes. One animal showed 2 strains among 2 pretreatment isolates and 1 animal showed 2 strains among 3 posttreatment isolates. Neither result affected cure decisions. Because of strain heterogeneity within herds, the number of observations per strain was too small for strain-specific assessment of cure, even though some strains were identified in multiple animals within herds (Table 3 ).

\section{Bacteriological Cure}

Across herds and species, $38 \%$ of control cows and $73 \%$ of the treated cows showed a bacteriological cure (Table 1). Important differences were observed between herds. The difference between treated and control animals was largest in herd $\mathrm{B}$ with a 63 percentage point increase in cure and was smallest in herd $\mathrm{E}$ with an increase of 19 percentage points (Table 1). To be considered a bacteriological cure, both posttreatment samples needed to be present. This was the case in $36(75 \%)$ of the control animals and in $50(89 \%)$ of

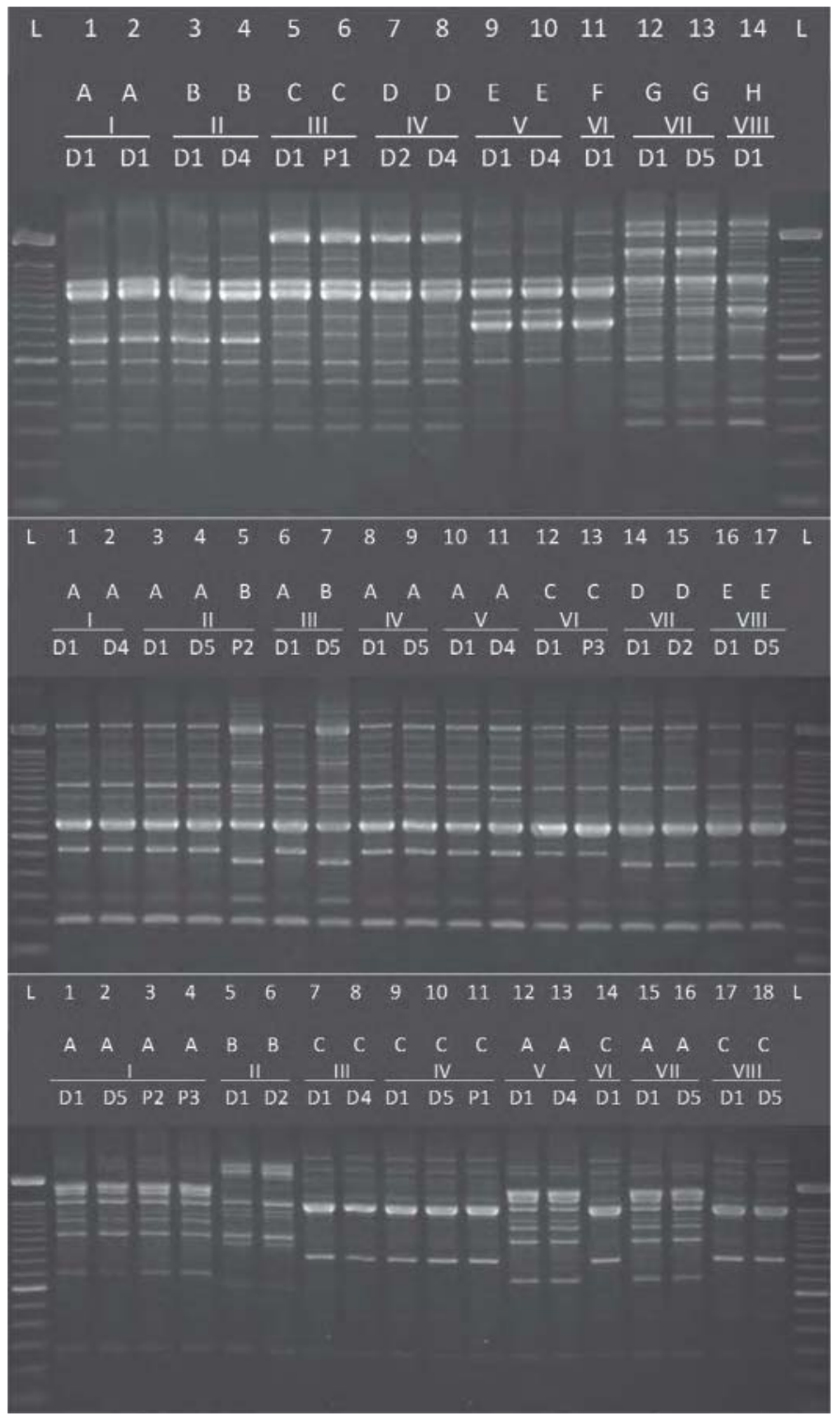

Figure 1. Examples of random amplification of polymorphic DNA (RAPD) typing results for 8 cows with Escherichia coli (top panel; herd E), Klebsiella pneumoniae (middle panel; herd C), or Enterobacter cloacae (bottom panel; herd A). Letters indicate strains (A through $\mathrm{H})$ or DNA ladder (L) and should be compared within panels only; roman numerals indicate cows; D1, D2, D4, and D5 indicate d 1, 2, 4 , and 5 of treatment, respectively; P1, P2, and P3 indicate d 1, 2, and 3 posttreatment, respectively. Strains are persistent within udder quarters (no cures) but may be different between cows for E. coli and E. cloacae. In the Klebsiella example, cow II would be considered cured (different strains pre- and posttreatment) whereas cow VI would be considered not cured (same strain pre- and posttreatment). 
Table 3. Distribution of random amplification of polymorphic DNA (RAPD) types among coliform species within herds

\begin{tabular}{llrrl}
\hline & & \multicolumn{2}{c}{ Number } & \\
\cline { 3 - 4 } Herd & Species & Isolates & Strains & Strain frequency $^{1}$ \\
\hline A & Enterobacter cloacae & 15 & 3 & $9,5,1$ \\
& Klebsiella spp. & 16 & 10 & $6,2,1,1,1,1,1,1,1,1$ \\
& Escherichia coli & 2 & 2 & 1,1 \\
$\mathrm{~B}$ & Klebsiella spp. & 10 & 8 & $2,2,1,1,1,1,1,1^{2}$ \\
& E. coli & 18 & 9 & $5,3,3,2,1,1,1,1,1$ \\
$\mathrm{C}$ & Klebsiella spp. & 5 & 4 & $2,1,1,1$ \\
& E. coli & 3 & 3 & $1,1,1$ \\
$\mathrm{D}$ & Klebsiella spp. & 1 & 1 & 1 \\
& E. coli & 17 & 10 & $5,2,2,2,1,1,1,1,1,1$ \\
\hline
\end{tabular}

${ }^{1}$ Number of isolates per RAPD type in descending frequency. For example, of E. cloacae isolates in herd A, 9 showed the most common RAPD type, 5 showed the second most common RAPD type, and 1 showed the third RAPD type.

${ }^{2}$ One isolate was identified as Klebsiella oxytoca; all other Klebsiella isolates in all farms were identified as Klebsiella pneumoniae.

the treated animals. When no or only one sample was available, the animals were classified as a bacteriological failure. Bacteriological cure was highest in $E$. coli-infected cows with $73 \%$. Bacteriological cure was $61 \%$ in E. cloacae-infected cows and $44.7 \%$ in Klebsiella spp.-infected cows. Figure 2 shows the least squares means of bacteriological cure by treatment group and pathogen.

Logistic regression results are shown in Table 4 . Logistic regression analysis was performed across all farms and across the 3 pathogens. This logistic regres- sion analysis showed a significant treatment effect $(P$ $=0.0004)$ with an odds ratio of 9.2 (95\% CI: 2.5-33.6). When the logistic regression analysis for E. coli was performed separately, ceftiofur treatment was statistically significant $(P=0.007)$, with an odds ratio of 112 (95\% CI: 2.8-397.3). For Klebsiella spp., treatment with ceftiofur was also significantly better compared with no treatment $(P=0.02)$ with an odds ratio of 8.5 (95\% CI: 1.1-30.1). For the E. cloacae-infected quarters, treatment was not significantly different from the nontreated controls $(P=0.39)$.

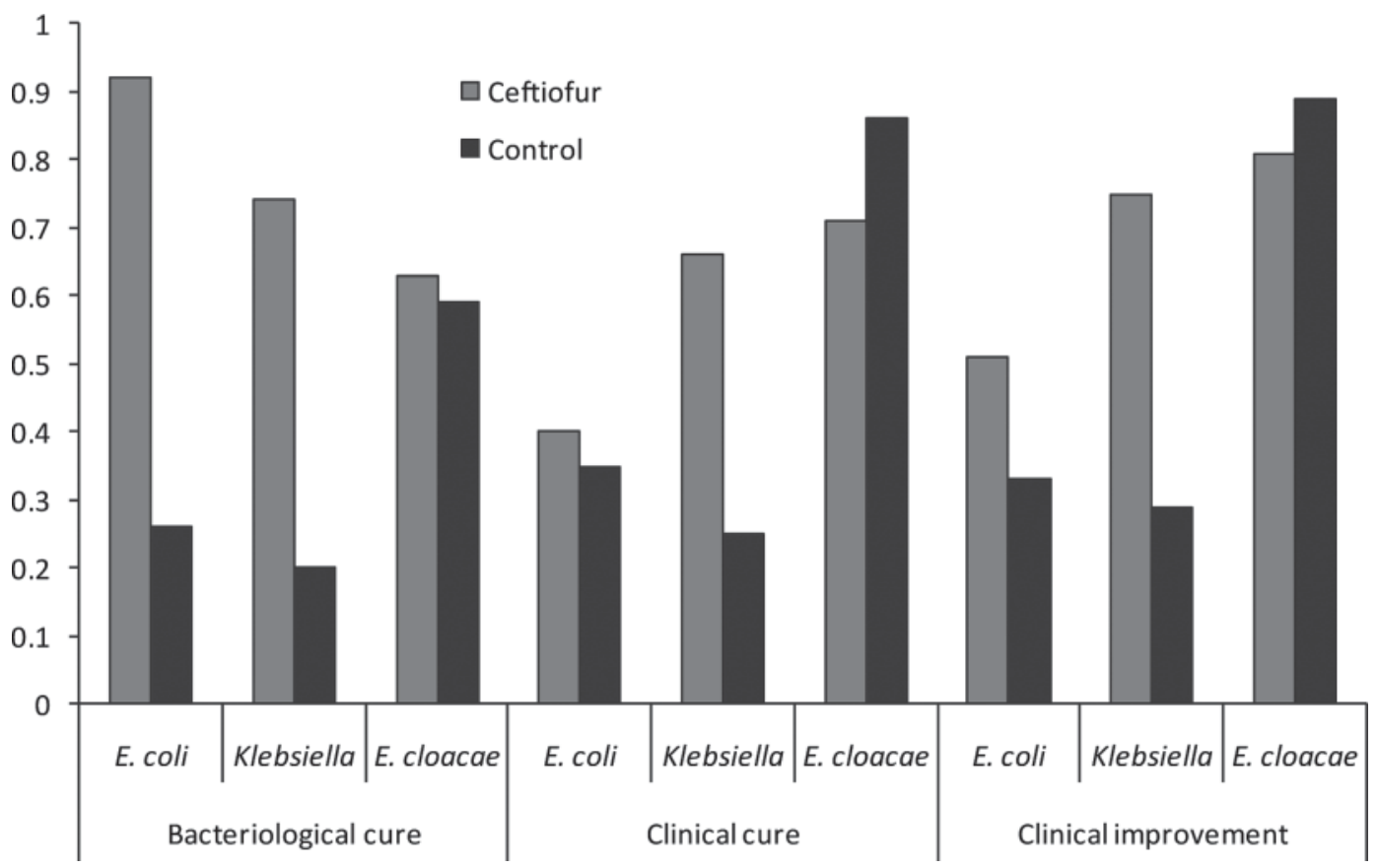

Figure 2. Least squares means by bacterial species of the final logistic regression models of bacteriological cure, clinical cure, and clinical improvement. $E$. coli $=$ Escherichia coli E. cloacae $=$ Enterobacter cloacae . 
Table 4. Results of logistic regression analysis of bacteriological cure; the final models for all cases, Escherichia coli, and Klebsiella spp. cases are shown ${ }^{1}$

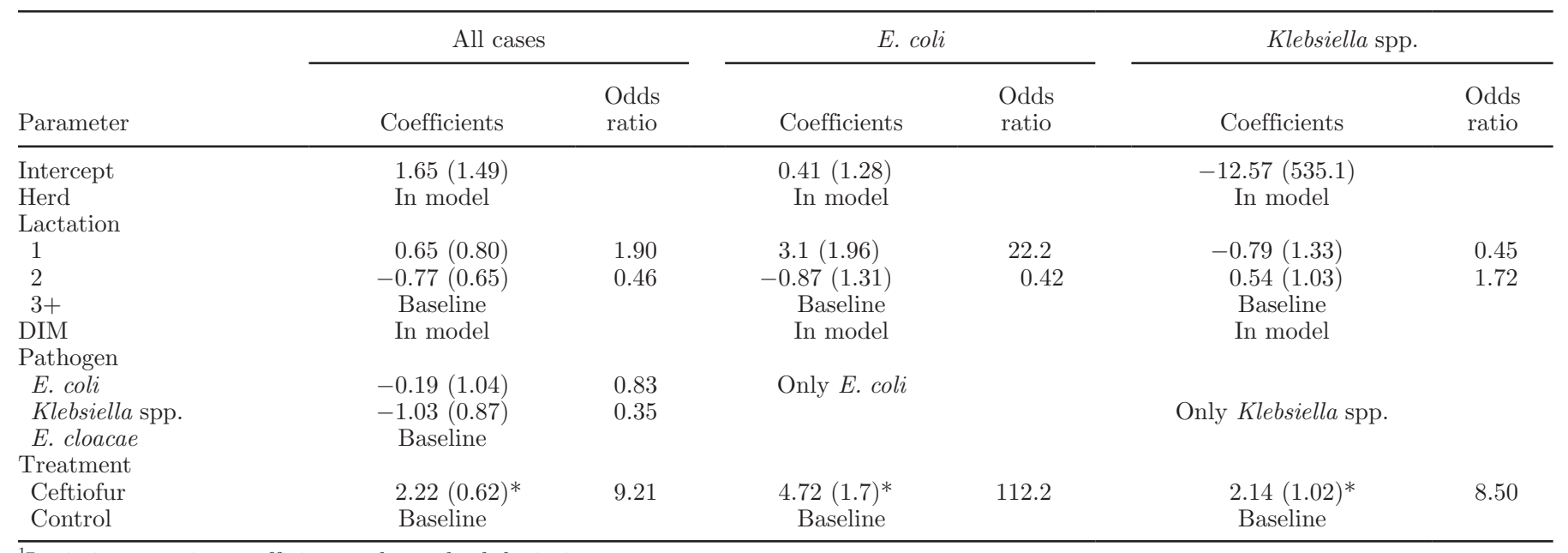

${ }^{1}$ Logistic regression coefficient and standard deviation.

$* P<0.05$.

\section{Clinical Cure and Clinical Improvement}

Clinical cure was defined as the complete absence of any clinical mastitis sign on both posttreatment sampling days; presence of any sign was considered a clinical failure. Of the cows that were defined as a clinical failure, 18 did not have data for both posttreatment days, 16 had watery milk, 15 still showed some evidence of clots or flakes, 12 had a swollen quarter, and 2 showed systemic signs of illness. Among all cows, $50 \%$ showed full clinical cure at both posttreatment sampling days. Proportion of clinical cure was $46 \%$ for control cows versus $54 \%$ for treated cows. Distribution of clinical cure among causal coliform bacteria and by treatment group is shown in Figure 2. The proportion of clinical cure was highest in cows infected with E. cloacae $(72 \%)$ and lowest in cows infected with E. coli $(42 \%)$. Logistic regression analysis on clinical cure, corrected for farm, lactation number, and DIM, showed an increased odds of cure for treated animals (odds ratio 9.2, 95\% CI: $2.5-33.6 ; P<0.01)$. In the logistic regression model, both E. coli- and Klebsiella spp.-infected cows were less likely to show clinical cure compared with the E. cloacae-infected cows, but this was not statistically significant. Clinical improvement (reduction of clinical score by at least $50 \%$ ) was present in $63 \%$ of treated cows and in $50 \%$ of control cows. The logistic regression analysis showed a significantly lower clinical improvement in animals infected with E. coli and Klebsiella spp. compared with cows infected with E. cloacae (the odds ratio were 0.16 and 0.24 , respectively; $P<0.05$ for both). Treated cows were significantly more likely to show clinical improvement compared with controls (odds ratio 2.4; $P<0.05$ ).

\section{Milk Production and SCC}

A relatively small number of cows had milk production data before and after clinical mastitis. In total, 29 of the 48 control cows (60\%) and 32 of the 56 treated cows $(57 \%)$ had sufficient milk production data available. Production decreased considerably after a case of CM (Figure 3). Both treated and control cows lost approximately $5 \mathrm{~kg}$ in milk production as measured on the next test-day after the clinical case. No significant difference in milk production decrease or recovery was seen between treated and control cows. When cows that showed bacteriological cure were compared with cows that did not cure, a significant difference in milk production recovery was observed. At the second test-day after the case of CM, cured cows had milk production that was approximately $4 \mathrm{~kg}$ higher than that of noncured cows $(P<0.05$; Figure 3$)$.

Linear scores before and after treatment are shown in Figure 3. Because of many animals with incomplete data, only 36 animals ( 15 controls and 21 treated cows) that had at least 2 pre- and 2 posttreatment test-days with LS data contributed to this analysis. Linear score before clinical mastitis was lower in control cows compared with treated cows (2.6 vs. 3.6). Linear score in the first test-day after CM was 4.6 in treated cows compared with 3.3 in control cows. In both groups, 

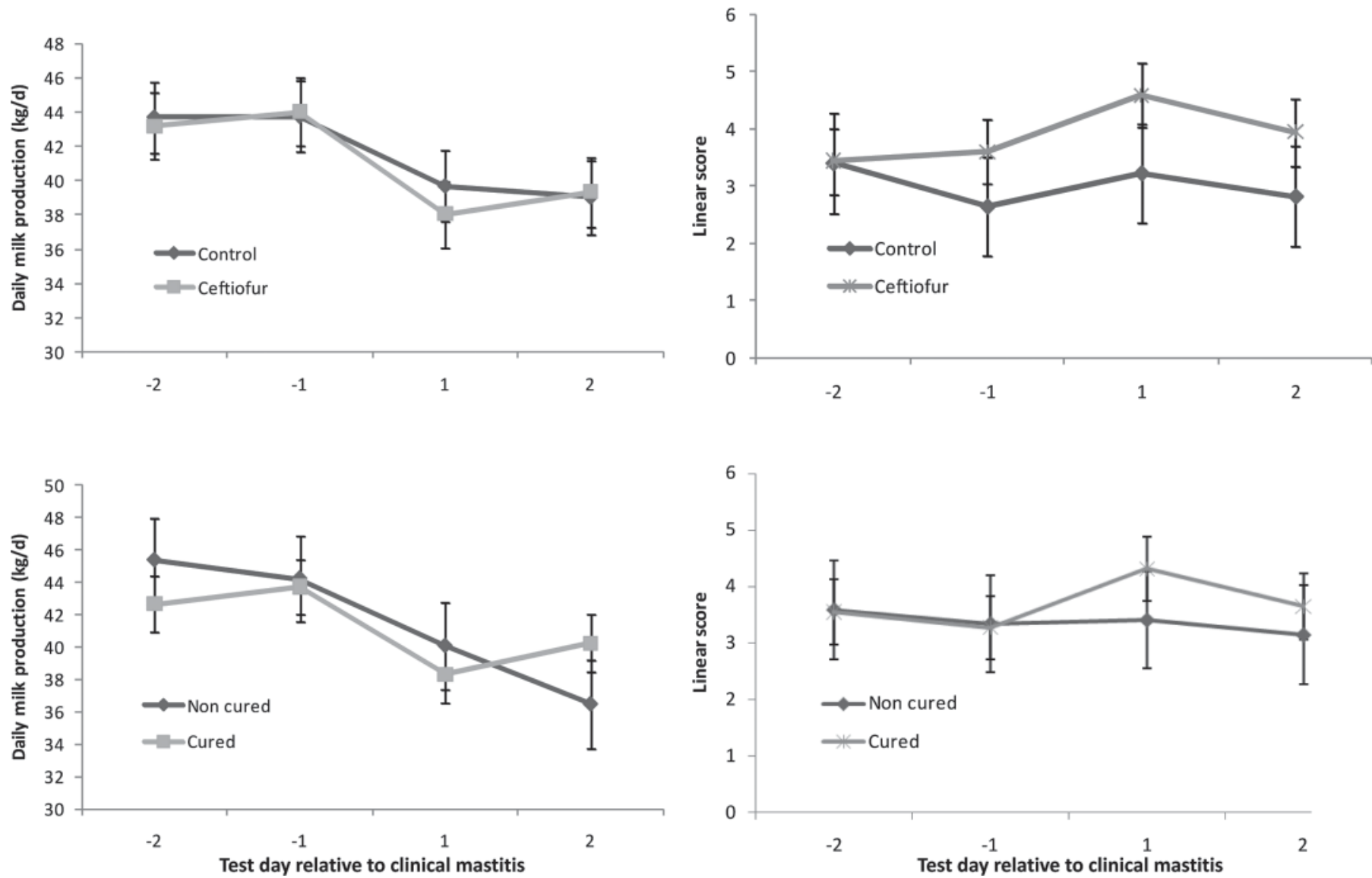

Figure 3. Least squares means of the final models for milk production (left) and linear score (right) before and after treatment in treated and control cows (top) and in bacteriologically cured and noncured animals (bottom). Sixty-one cows were included in the milk production analysis (32 treated, 29 control) and 36 in the linear score analysis (21 treated, 15 control).

LS dropped thereafter to 3.9 and 2.8, respectively. No significant differences were found in LS either before or after treatment. Cows with bacteriological cure showed statistically nonsignificant but higher LS in the first test-day after CM (4.3 in cured animals vs. 3.4 in noncured animals).

\section{Exit from the Study and Exit from the Herd}

Twenty-three animals were removed from the study early. Of these 23 animals, 13 were in the control group (13 of 48, 27\%) and 10 were in the treated group (10 of $56,18 \%)$. Early culling from the herd or death occurred in 16 animals, 9 of which were control animals (19\%) and 7 of which were treated animals (12\%). Figure 4 shows the survival curves for time to exit from the study and time to exit from the herd. Survival curves are shown for treated versus control cows and for bacteriologically cured versus noncured cows. Cox proportional hazard models showed a significant difference in time to exit from the study between treated and control cows. The hazard ratio of exit from the study between treated versus control cows was 0.25 (95\% CI: $0.09-0.67 ; P$ $<0.05)$. Cows that were bacteriologically cured had a hazard ratio of exit from the study compared with noncured cows of 0.04 (95\% CI: $0.01-0.21 ; P<0.05$ ). The hazard ratio of exit from the herd in treated versus control cows was 0.62 (95\% CI: $0.19-1.97 ; P>0.2$ ). In bacteriologically cured versus noncured cows the hazard ratio was 0.19 (95\% CI: 0.05-0.68; $P<0.05)$.

\section{DISCUSSION}

Across farms, animals in treatment and control groups did not differ significantly in parity, lactation stage, milk production, or SCC at the time of enrollment. However, after the occurrence of clinical coliform mastitis, significant and important differences were observed between treated and control groups. Across farms and coliform species, 5-d treatment with ceftiofur resulted in a significantly higher probability of cure compared with no treatment. Across herds and 

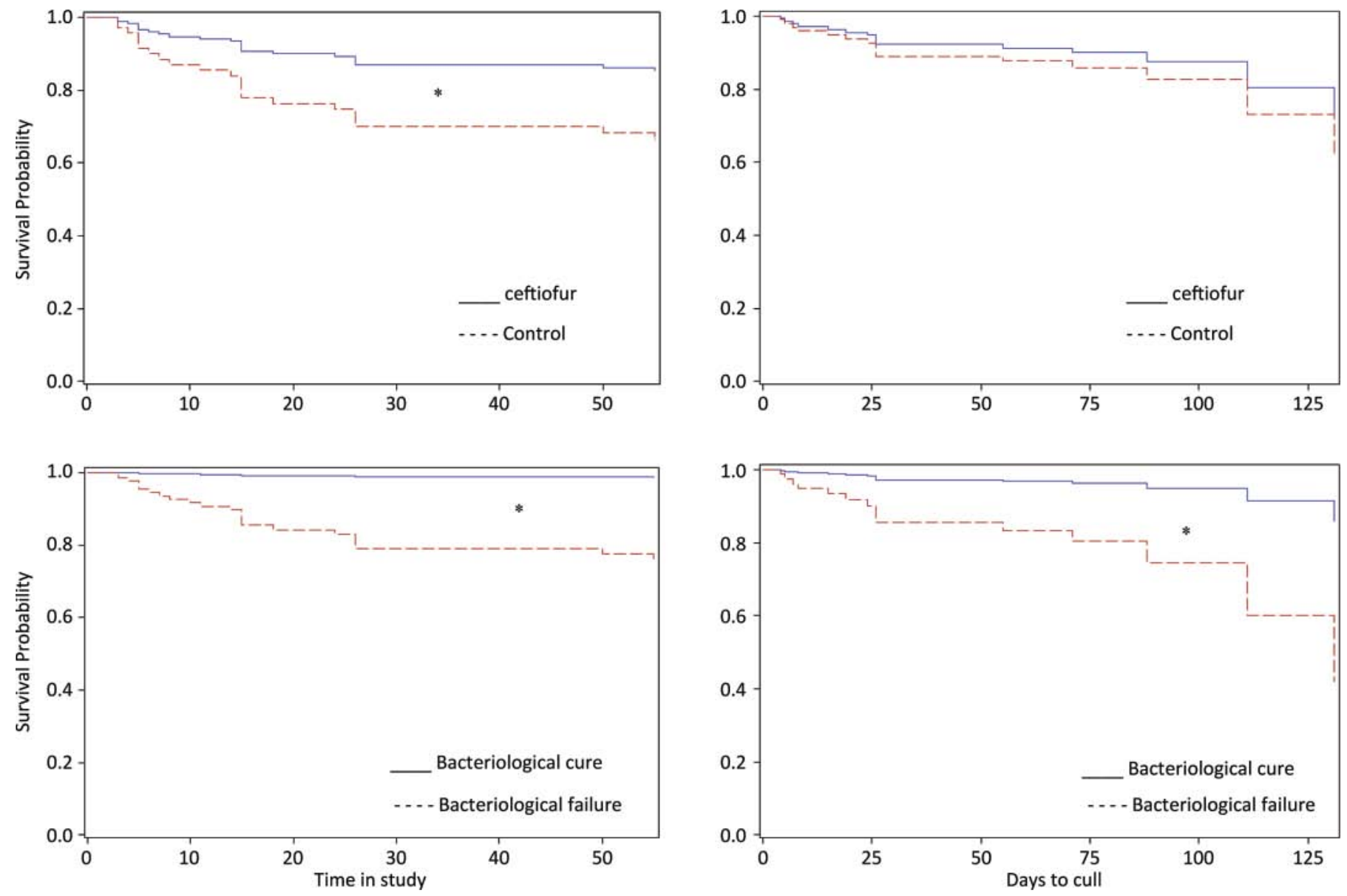

Figure 4. Survival curves for time to exit from study (left) and time to culling (right). A comparison of ceftiofur-treated animals with controls is shown (top) and animals with bacteriological cure versus noncure (bottom). *Indicates significant difference in curves based on Cox regression analysis, $P<0.05$. Color version available in the online PDF.

bacteriological species, bacteriological cure was $73 \%$ in treated animals and $38 \%$ in control animals. For quarters infected with E. coli, bacteriological cure was $89 \%$ in treated animals versus $53 \%$ in control animals, and for quarters infected with Klebsiella, bacteriological cure was $57 \%$ in treated animals versus $19 \%$ in control animals. Quarters infected with E. cloacae showed no difference in bacteriological cure between treated and control animals, although this finding was based on a relatively small sample. Although E. cloacae IMI are not generally reported, they have been observed as a cause of clinical mastitis (Nam et al., 2009).

This study is one of very few field studies showing a significant effect of antibiotic treatment on clinical coliform mastitis. The special characteristics of this study were the inclusion of only mild and moderate cases of mastitis and the extended duration of intramammary therapy. These characteristics may have contributed to the improved cure rate. However, the study was also characterized by a nonblinded design. Dairy farmers were aware that cows were either treated or not treated and farmer intervention was specifically allowed in the trial design. Thus, some farmer bias toward early intervention in control cows may have occurred. Cows that left the study due to intervention by the dairy farmer were scored as noncures, resulting in a potentially biased efficacy of treatment. We would argue, however, that this bias is minor because the current treatment protocol for clinical mastitis due to coliform infections in many dairy farms (including the farms in our study) is no intramammary antibiotic treatment at all. Hence, the control group was the treatment of choice for the farms at the start of the study.

This study showed a significant beneficial effect of ceftiofur intramammary treatment on bovine coliform mastitis. Although little antimicrobial resistance against this third-generation cephalosporin is currently present in isolates from bovine mastitis cases, it would be prudent to continue to monitor for development of resistance in these isolates and commensal species from on-farm sources. In human hospital settings, increased use of extended-spectrum $\beta$-lactamases has reduced the 
efficacy of this class of antibiotics (Pfeifer et al., 2010), and this resistance has recently been reported in bovine mastitis pathogens (Locatelli et al., 2009). An increased resistance to third-generation cephalosporin would be of potential concern for both veterinary and human applications.

Spontaneous cure rates of E. coli infections are generally high. For example, Leininger and coworkers (2003) observed that almost all mild and moderate $\mathrm{CM}$ cases due to E. coli cured within a short time frame, regardless of whether antimicrobial treatment or frequent milk-out was used. In that study, $85 \%$ bacteriological cure was observed within $7 \mathrm{~d}$ of $\mathrm{CM}$. In the current study, however, $47 \%$ of infections in untreated control quarters did not meet the definition for bacteriological cure within the 2 -wk follow-up period. The reason for this low percentage of spontaneous cure is likely 2-fold. First, cows did not have complete follow-up data, which we defined as a failure (3 out of the 10 noncures in the control group) and second, cows were truly not cured and a persistent $E$. coli infection was present (7 out of the 10 noncures in the control group). Persistent $E$. coli mastitis is increasingly recognized as a clinically relevant problem with a distinct pathogenesis (Dogan et al., 2006; White et al., 2010; Suojala et al., 2011).

Quarters infected with Klebsiella spp. showed a relatively high probability of bacteriological cure in the treatment group (57\%). A similar proportion of $50 \%$ cure was observed after intramuscular ceftiofur treatment of 8 animals with severe CM (Erskine et al., 2002a), but clearly the number of quarters in that study was small. Quarters infected with Klebsiella are often considered to have a very low chance of cure. In a study by Roberson and colleagues (2004), 37\% of 19 Klebsiella-infected quarters from cows with mild to moderate CM cured within a week, and $47 \%$ cured within 36 d. In the Erskine et al. (2002a) study of severe CM, only 1 of 4 control cows cured. In our study, control quarters infected with Klebsiella spp. had a very low probability of spontaneous cure (19\%), and the improvement in bacteriological cure after $5 \mathrm{~d}$ of antimicrobial treatment with intramammary ceftiofur was statistically significant compared with the nontreated control group.

Our data from 5 dairy herds in New York State suggest that distinct bacteriological cure profiles exist for the different coliform bacterial species. This different cure profile across bacterial species makes it important to determine, at least at the herd level, which type of coliform is the predominant cause of clinical mastitis in the herd. In herds where E. cloacae is dominant, treatment is unlikely to result in any improvements, whereas in herds with a dominance of E. coli and Klebsiella spp., treatment with ceftiofur hydrochloride for $5 \mathrm{~d}$ appears to be efficacious.

Coliform mastitis is associated with significant losses in milk production that may persist for weeks, particularly in the case of Klebsiella infections, which were reported to cause average losses of $7.6 \mathrm{~kg} / \mathrm{d}$ shortly after infection and $5 \mathrm{~kg} / \mathrm{d}$ in subsequent months (Gröhn et al., 2004). In our data, when compared with milk production before mastitis, cows lost approximately 10 $\mathrm{kg}$ of milk per day with no bacteriological cure and $4 \mathrm{~kg}$ of milk per day when there was a bacteriological cure (Figure 3, second test-day after treatment). When quarters were cured of the microorganism that was cultured at the time of diagnosis of $\mathrm{CM}$, cows responded with a significantly higher milk production after completion of treatment. An approximately $6-\mathrm{kg}$ increase in daily milk production is an important benefit for the producer investing in antimicrobial therapy, additional labor, and the treatment-associated milk discards. Clearly, not every treatment resulted in cure and not every untreated cow failed to cure; however, the probability of cure approximately doubled after treatment. Although LS was numerically lower in cured cows, no significant difference was observed posttreatment between cured and noncured quarters or between treated and control quarters. This was likely due to the large variability within groups and the relatively small number of cows that contributed to the analyses ( $\mathrm{n}=$ 36 out of 104 cows).

Herd survival was significantly higher in bacteriologically cured animals versus noncured animals. Because a cow that was culled before both treatment samples were taken was defined as a noncure, it is not surprising to see a relationship between the rate of culling and noncure.

Ultimately, the value of treatment needs to be decided based on economic arguments. Precise data, preferably from field trials, are essential in the development of valid economic models. The data in this study will be helpful in future developments of economic models on treatment decisions for gram-negative mild and moderate clinical mastitis. The use of a third-generation cephalosporin for bovine mastitis is currently under debate. It may be argued that intramammary use of this antimicrobial is preferable over systemic use with regard to emergence of antimicrobial resistance in microbiota of the gastrointestinal tract, respiratory mucosa, or skin. A quantification of the risk or emergence of resistance after parenteral versus intramammary use of ceftiofur hydrochloride would be of considerable value in the discussion on rational treatment options for bovine mastitis.

In this study, we used genotypic strain typing as the gold standard for bacteriological cure. The use of geno- 
typing of bacteria further refines the definition of cure, as previously shown for Staphylococcus aureus mastitis (Luby and Middleton, 2005). This definition takes advantage of current diagnostic technologies (Zadoks and Schukken, 2006), but assumes that quarters are infected with a single or at least a single dominant bacterial strain. Several studies of gram-positive and gram-negative mastitis show that this is generally the case (Oliver et al., 1998; Phuektes et al., 2001; Young et al., 2001; Munoz et al., 2007), although exceptions have been described for Klebsiella spp. (Paulin-Curlee et al., 2007). In the latter study, assessment of reproducibility of typing results was not described, whereas we demonstrated that our method had good reproducibility. We observed dominant strain types for all 3 bacterial species in at least some of the farms. The results challenge the paradigm that gram-negative infections are strictly environmental and suggest that at least some of gram-negative infections may be due to cow-to-cow transmission or point-source exposure to a common strain (Zadoks and Schukken, 2006; Munoz et al., 2007). To reduce the possibility that lack of discriminatory power explained the presence of the same strain in multiple animals, a selection of strains was tested by an alternative RAPD protocol that has been used in several peer-reviewed studies of E. coli and Klebsiella spp. (Lam et al., 1996; Bradley and Green, 2000; Munoz and Zadoks, 2007). As before (Munoz and Zadoks, 2007), the alternative RAPD protocol did not increase the number of strains detected.

\section{CONCLUSIONS}

Based on a randomized, controlled clinical trial in 5 dairy herds with evaluation of production and clinical data and bacteriological culture results, treatment of nonsevere clinical gram-negative mastitis with ceftiofur hydrochloride resulted in significant bacteriological improvement compared with nontreated control animals, particularly in animals infected with E. coli or Klebsiella spp. Treated animals clinically improved significantly more compared with control cows. No significant differences were observed in milk production or linear score either before or after clinical mastitis between treated and control animals. Treated animals left the study less frequently compared with control animals.

\section{ACKNOWLEDGMENTS}

We acknowledge the support of the 5 New York State dairy farmers participating in this study. We acknowledge the Northern New York Agricultural Development Program and Pfizer Inc. (New York, NY) for providing financial and in-kind support for this study.

\section{REFERENCES}

Barkema, H. W., Y. H. Schukken, T. J. G. M. Lam, M. L. Beiboer, H. Wilmink, G. Benedictus, and A. Brand. 1998. Incidence of clinical mastitis in dairy herds grouped in three categories by bulk milk somatic cell counts. J. Dairy Sci. 81:411-419.

Bengtsson, B., H. E. Unnerstad, T. Ekman, K. Artursson, M. NilssonOst, and K. P. Waller. 2009. Antimicrobial susceptibility of udder pathogens from cases of acute clinical mastitis in dairy cows. Vet. Microbiol. 136:142-149.

Bradley, A. J., and M. J. Green. 2000. A study of the incidence and significance of intramammary enterobacterial infections acquired during the dry period. J. Dairy Sci. 83:1957-1965.

Breen, J. E., M. J. Green, and A. J. Bradley. 2009. Quarter and cow risk factors associated with the occurrence of clinical mastitis in dairy cows in the United Kingdom. J. Dairy Sci. 92:2551-2561.

Dogan, B., S. Klaessig, M. Rishniw, R. A. Almeida, S. P. Oliver, K. Simpson, and Y. H. Schukken. 2006. Adherent and invasive Escherichia coli are associated with persistent bovine mastitis. Vet. Microbiol. 116:270-282.

Erskine, R. J., P. C. Bartlett, J. L. VanLente, and C. R. Phipps. 2002a. Efficacy of systemic ceftiofur as a therapy for severe clinical mastitis in dairy cattle. J. Dairy Sci. 85:2571-2575.

Erskine, R. J., R. J. Eberhart, L. J. Hutchinson, S. B. Spencer, and M. A. Campbell. 1988. Incidence and types of clinical mastitis in dairy herds with high and low somatic cell counts. J. Am. Vet. Med. Assoc. 192:761-765.

Erskine, R. J., R. D. Walker, C. A. Bolin, P. C. Bartlett, and D. G. White. 2002b. Trends in antibacterial susceptibility of mastitis pathogens during a seven-year period. J. Dairy Sci. 85:1111-1118.

Erskine, R. J., R. C. Wilson, J. W. Tyler, K. A. McClure, R. S. Nelson, and H. J. Spears. 1995. Ceftiofur distribution in serum and milk from clinically normal cows and cows with experimental Escherichia coli-induced mastitis. Am. J. Vet. Res. 56:481-485.

FDA. 2005. Approved animal drug producrts. Accessed Oct, 3, 2011 http://www.fda.gov/downloads/AnimalVeterinary/Products/ ApprovedAnimalDrugProducts/FOIADrugSummaries/ucm 118051.pdf.

Gröhn, Y. T., D. J. Wilson, R. N. González, J. A. Hertl, H. Schulte, G. Bennett, and Y. H. Schukken. 2004. Effect of pathogen-specific clinical mastitis on milk yield in dairy cows. J. Dairy Sci. 87:3358-3374

Hoeben, B. D., E. Monfardini, C. Burvenich, and J. Hamann. 2000. Treatment of acute Escherichia coli mastitis in cows with enrofloxacin: Effect on clinical signs and chemiluminescence of circulating neutrophils. J. Dairy Res. 67:485-502.

Hogan, J., and K. L. Smith. 2003. Coliform mastitis. Vet. Res. 34:507-519.

Lam, T. J., L. J. Lipman, Y. H. Schukken, W. Gaastra, and A. Brand. 1996. Epidemiological characteristics of bovine clinical mastitis caused by Staphylococcus aureus and Escherichia coli studied by DNA fingerprinting. Am. J. Vet. Res. 57:39-42.

Leininger, D. J., J. R. Roberson, F. Elvinger, D. Ward, and R. M. Akers. 2003. Evaluation of frequent milkout for treatment of cows with experimentally induced Escherichia coli mastitis. J. Am. Vet. Med. Assoc. 222:63-66.

Locatelli, C., I. Caronte, L. Scaccabarozzi, R. Migliavacca, L. Pagani, and P. Moroni. 2009. Extended-spectrum beta-lactamase production in E. coli strains isolated from clinical bovine mastitis. Vet. Res. Commun. 33(Suppl. 1):141-144.

Luby, C. D., and J. R. Middleton. 2005. Efficacy of vaccination and antibiotic therapy against Staphylococcus aureus mastitis in dairy cattle. Vet. Rec. 157:89-90.

Munoz, M. A., F. L. Welcome, Y. H. Schukken, and R. N. Zadoks. 2007. Molecular epidemiology of two Klebsiella pneumoniae outbreaks on a dairy farm in New York State. J. Clin. Microbiol. 45:3964-3971.

Munoz, M. A., and R. N. Zadoks. 2007. Short communication: Patterns of fecal shedding of Klebsiella by dairy cows. J. Dairy Sci. 90:1220-1224. 
Nam, H. M., S. K. Lim, H. M. Kang, J. M. Kim, J. S. Moon, K. C. Jang, J. M. Kim, Y. S. Joo, and S. C. Jung. 2009. Prevalence and antimicrobial susceptibility of gram-negative bacteria isolated from bovine mastitis between 2003 and 2008 in Korea. J. Dairy Sci. 92:2020-2026.

National Mastitis Council. 1999. Laboratory Handbook on Bovine Mastitis. National Mastitis Council, Madison, WI.

Olde Riekerink, R. G., H. W. Barkema, D. F. Kelton, and D. T. Scholl. 2008. Incidence rate of clinical mastitis on Canadian dairy farms. J. Dairy Sci. 91:1366-1377.

Oliver, S. P., B. E. Gillespie, and B. M. Jayarao. 1998. Detection of new and persistent Streptococcus uberis and Streptococcus dysgalactiae intramammary infections by polymerase chain reactionbased DNA fingerprinting. FEMS Microbiol. Lett. 160:69-73.

Paulin-Curlee, G. G., R. S. Singer, S. Sreevatsan, R. Isaacson, J. Reneau, D. Foster, and R. Bey. 2007. Genetic diversity of mastitis-associated Klebsiella pneumoniae in dairy cows. J. Dairy Sci. 90:3681-3689

Pfeifer, Y., A. Cullik, and W. Witte. 2010. Resistance to cephalosporins and carbapenems in gram-negative bacterial pathogens. Int. J. Med. Microbiol. 300:371-379.

Phuektes, P., P. D. Mansell, R. S. Dyson, N. D. Hooper, J. S. Dick, and G. F. Browning. 2001. Molecular epidemiology of Streptococcus uberis isolates from dairy cows with mastitis. J. Clin. Microbiol. 39:1460-1466.

Poutrel, B., M. R. Stegemann, O. Roy, F. Pothier, N. Tilt, and M. Payne-Johnson. 2008. Evaluation of the efficacy of systemic danofloxacin in the treatment of induced acute Escherichia coli bovine mastitis. J. Dairy Res. 75:310-318.

Pyörälä, S., L. Kaartinen, H. Käck, and V. Rainio. 1994. Efficacy of two therapy regimens for treatment of experimentally induced Escherichia coli mastitis in cows. J. Dairy Sci. 77:453-461.

Rantala, M., L. Kaartinen, E. Välimäki, M. Stryrman, M. Hiekkaranta, A. Niemi, L. Saari, and S. Pyörälä. 2002. Efficacy and pharmacokinetics of enrofloxacin and flunixin meglumine for treatment of cows with experimentally induced Escherichia coli mastitis. J. Vet. Pharmacol. Ther. 25:251-258.

Roberson, J. R., L. D. Warnick, and G. Moore. 2004. Mild to moderate clinical mastitis: Efficacy of intramammary amoxicillin, frequent milk-out, a combined intramammary amoxicillin, and frequent milk-out treatment versus no treatment. J. Dairy Sci. 87:583-592.
Shpigel, N. Y., D. Levin, M. Winkler, A. Saran, G. Ziv, and A Böttner. 1997. Efficacy of cefquinome for treatment of cows with mastitis experimentally induced using Escherichia coli. J. Dairy Sci. 80:318-323.

Srinivasan, V., B. E. Gillespie, M. J. Lewis, L. T. Nguyen, S. I. Headrick, Y. H. Schukken, and S. P. Oliver. 2007. Phenotypic and genotypic antimicrobial resistance patterns of Escherichia coli isolated from dairy cows with mastitis. Vet. Microbiol. 124:319-328.

Suojala, L., T. Pohjanvirta, H. Simojoki, A. L. Myllyniemi, A. Pitkälä, S. Pelkonen, and S Pyörälä.. 2011. Phylogeny, virulence factors and antimicrobial susceptibility of Escherichia coli isolated in clinical bovine mastitis. Vet. Microbiol. 147:383-388.

Suojala, L., H. Simojoki, K. Mustonen, L. Kaartinen, and S. Pyörälä. 2010. Efficacy of enrofloxacin in the treatment of naturally occurring acute clinical Escherichia coli mastitis. J. Dairy Sci. 93:19601969.

Todhunter, D. A., K. L. Smith, J. S. Hogan, and P. S. Schoenberger. 1991. Gram-negative bacterial infections of the mammary gland in cows. Am. J. Vet. Res. 52:184-188.

Wenz, J. R., G. M. Barrington, F. B. Garry, R. P. Dinsmore, and R. J. Callan. 2001. Use of systemic disease signs to assess disease severity in dairy cows with acute coliform mastitis. J. Am. Vet. Med. Assoc. 218:567-572

Wenz, J. R., F. B. Garry, and G. M. Barrington. 2006. Comparison of disease severity scoring systems for dairy cattle with acute coliform mastitis. J. Am. Vet. Med. Assoc. 229:259-262.

Wenz, J. R., F. B. Garry, J. E. Lombard, R. Elia, D. Prentice, and R. P. Dinsmore. 2005. Efficacy of parenteral ceftiofur for treatment of systemically mild clinical mastitis in dairy cattle. J. Dairy Sci. 88:3496-3499

White, L. J., Y. H. Schukken, B. Dogan, L. Green, D. Döpfer, M. J. Chappell, and G. F. Medley. 2010. Modelling the dynamics of intramammary $E$. coli infections in dairy cows: Understanding mechanisms that distinguish transient from persistent infections. Vet. Res. 41:13-21.

Young, B., D. Platt, D. Logue, H. Ternent, and J. Fitzpatrick. 2001. Bovine Staphylococcus aureus mastitis: strain recognition and dynamics of infection. J. Dairy Res. 68:377-388.

Zadoks, R. N., and Y. H. Schukken. 2006. Use of molecular epidemiology in veterinary practice. Vet. Clin. North Am. Food Anim. Pract. 22:229-261. 